\title{
Utilisation of voluntary counselling and testing services among bar waitresses in Kinondoni District, Dar es Salaam, Tanzania
}

\author{
ANNA TENGIA-KESSY** and DOMINICA E. LYAMUYA ${ }^{2}$ \\ ${ }^{1}$ School of Public Health and Social Sciences, Muhimbili University of Health and Allied Sciences, P. O. Box 65015, Dar \\ es Salaam, Tanzania \\ ${ }^{2}$ Management for Development in Health, P.O. Box 65540, Dar es Salaam, Tanzania
}

\begin{abstract}
Background: Tanzania is among sub-Saharan countries severely affected by the HIV and AIDS epidemic, with an increased burden among high-risk populations, such as bar waitresses. Voluntary counselling and testing (VCT) is among effective approaches to slow down the spread of HIV infection and minimize its impact. However, little information is available on the extent of utilisation of VCT services among bar waitresses. The study examined utilisation of VCT services and its influencing factors among bar waitresses in Kinondoni district, Dar es Salaam, Tanzania.

Methods: We used an interview schedule to gather information on VCT utilisation from 378 bar waitresses, identified using a multi-stage sampling technique. Data were analysed using the Statistical Package for Social Science (Version 15.0). A p-value less than 0.05 was used as a cut-off value for availability of a statistical association between VCT utilisation and related independent factors. Logistic regression models were employed to assess independent correlates of VCT use.

Results: The mean (standard deviation) age of the respondents was 26.1 (5.0) years. About $60 \%$ had never been married and about $70 \%$ had at least one child at the time of the survey. Majority of the bar waitresses (89.7\%) affirmed to have ever utilized VCT services at some point in their lifetime. Despite the high level of knowledge on VCT (71.4\%), the services were irregularly utilized with less than half ( $45.5 \%)$ of the respondents utilizing them within six months prior to the survey. Independent predictors for service utilisation included having a child, having higher knowledge on VCT, using a condom in the most recent sexual act as well as lack of fear of positive HIV results or stigmatization.

Conclusions: Utilisation of VCT services is high among bar waitresses in Kinondoni district. However, the services are not utilised regularly as recommended for high-risk populations. Findings call for comprehensive strategies to enhance effective service utilisation and further studies based on a broader and more inclusive sample size.
\end{abstract}

Keywords: bar waitress, determinants, HIV testing, counselling, Tanzania

\section{Introduction}

Acquired Immunodeficiency Syndrome (AIDS) has reached pandemic levels and remains the most serious public health problem globally. In 2016, estimates indicate that globally, 36.7 million people were living with HIV and around 30\% were not aware of their HIV sero-status (UNAIDS, 2017). Tanzania remains one of the sub-Saharan countries severely affected by the epidemic; with HIV prevalence of $5.1 \%$ among all adults aged $15-49$ years. The prevalence of HIV is generally higher among women (6.2\%) than among men (3.8\%). Women working in hotels, restaurants, bars and other food and recreational facilities have substantially higher HIV prevalence and incidence than women in the general population (Vallely et al., 2007; Watson-Jones et al., 2007). For example, the prevalence of HIV among female bar workers (bar waitresses) has been documented to be as high as $26.3 \%$ and $68 \%$ in Northern Tanzania and Mbeya region respectively (Kapiga et al., 2002; Riedner et al., 2003).

\footnotetext{
*Correspondence email: atengia@gmail.com
} 
Like many other countries, Tanzania has taken different approaches in the effort to slow the spread of HIV infection and reduce its impact (Charles et al., 2009; Addis et al., 2013). These approaches include voluntary counselling and testing (VCT); which has been internationally documented as an effective and important strategy for both prevention and care of HIV (Aho et al., 2011; Uzochukwu et al., 2011). Although members in the general population fail to regularly utilize VCT services due to several reasons (Obermeyer \& Osborn, 2007), uptake of VCT services in Tanzania has increased from $37 \%$ to $62 \%$ among women and from $27 \%$ to $47 \%$ among men in 2008 and 2012 respectively (THMIS, 2008, 2013). Encouraging early detection of HIV infection among high risk populations like bar waitresses through VCT is crucial because HIV testing is considered one of the important steps for controlling the epidemic. Testing is the entry point for prevention, care and treatment and support services (NACP, 2008).

Despite the increased number of VCT sites and the extensive campaigns to promote its utilisation through mass media, testing for HIV remains a challenge not only in Tanzania but globally. This challenge is remarkable in high-risk population groups where evidence shows that utilisation of the services is not as routinely as recommended (NACP, 2011). The Tanzania National Multi-Sectoral HIV Prevention Strategy of 2009-2012 recommends VCT for HIV as one of the measures in the minimum package of prevention services for most at risk populations, bar waitresses inclusive. However, little information is available on the uptake of VCT services for HIV among bar waitresses in Tanzania. Therefore this study was carried out to determine the utilisation of counselling and testing services among bar waitresses in Kinondoni District of eastern Tanzania.

\section{Materials and methods}

\section{Study area and design}

This analytical cross-sectional survey was conducted in selected wards of Kinondoni district, Dar es Salaam, Tanzania. Kinondoni is an urban district with four divisions and 34 wards. This district was purposively identified for the study since it has most of the registered bars in Dar es Salaam. This survey focused on bar waitresses (females working in bars and pubs, responsible for serving foods and drinks to customers) in Kinondoni district; available in their respective sites on the day of data collection. Sample size estimation was based on $69 \%$, proportion of female sex workers in Tanzania (NACP, 2010) utilizing VCT. This proportion was used because data on bar waitresses utilizing VCT are not available. It was hypothesized that these two groups share some HIV risks.

Using a $95 \%$ confidence interval, a precision of $5 \%$ and a design effect of 2, with a correction of $10 \%$ non-response rate, a minimum sample size of 371 bar waitresses was reached. A three-stage random cluster sampling design was employed, starting with selection of divisions, then wards in selected divisions and finally 10 bars from each of the wards. The sampling frames were obtained from respective ward secretaries. All available waitresses in these bars were invited to participate in the survey.

\section{Data collection}

Using a pre-tested interview schedule, trained research assistants conducted face-to-face interviews with the respondents after getting their informed consent. Each interview was conducted in a conducive and private place within the respective bar. The dependent variable of interest in this study was ever utilisation of HIV VCT whereas the independent variables were socio-demographic characteristics of the bar waitresses, knowledge on HIV VCT services; risk sexual practices, reasons for using VCT services and barriers for utilizing the VCT services. 


\section{Data analysis}

The data were checked for accuracy and completeness. The open-ended questions were coded and categorized based on the main emerging themes. Descriptive statistical measures like mean and standard deviations (SD) were derived for continuous variables. Frequencies were produced for categorical variables and association examined using chi-square test. Utilisation of VCT services was measured as ever use (the main outcome variable) or recent use (within six months before the survey). All statistical procedures were performed using the Statistical Package for Social Sciences (SPSS) version 15.0.

We measured knowledge about VCT based on five questions of different weights. Three of the questions carried one point each while the other two were assigned two points each. The first composite knowledge variable was created with values ranging between 0 and 7 . On the other hand, the second composite variable constituted of 1 for respondents who mentioned two places that offer VCT services and two benefits of the services; and o otherwise. The knowledge levels were categorized as 'high'; 'moderate' or 'low' if the scores were respectively 6-8, 3-5 or 0-2.

Unadjusted and adjusted logistic regression models were produced with VCT utilisation and the potential determinants as the outcome and independent variables respectively. All factors with $p<0.20$ in the unadjusted analyses were included in the multivariable model.

\section{Ethical considerations}

Ethical approval to conduct this study was granted by the Research Ethics Committee, Muhimbili University of Health and Allied Sciences. Permissions to collect data were requested and granted from the regional, district and respective ward authorities and managers of the selected bars. Ethical issues specific for this study that needed attention included the fact that participants were asked to disclose sensitive information relating to their sexual practices, which could elicit emotional feelings. To address this, participants were interviewed in an area that ensured privacy and they were assured of confidentiality of any information that they shared with the research team. Informed consent was sought from each respondent before administering the survey tool. Each potential study participant was asked to sign (or thump-sign) the consent form. At the end of the interview, respondents who had not accessed VCT services were encouraged to use them.

\section{Results}

\section{Background characteristics of the study participants}

A total of 378 bar waitresses participated in this study, their ages ranged from 17 to 54 years with a mean of 26.1 ( $S D=5.0$ ) years. Slightly more than half, $195(51.6 \%)$, of the respondents were in the age group 25-34 years and 221 (58.5\%) reported never married (Table 1). The majority, 350 (95.0\%), of these bar waitresses had attended at least some primary school education. Furthermore, a third, 114 (30.2\%), of the respondents reported that they never had a child by the time of the survey.

Table 1. Background characteristics of the study participants $(n=378)$

\begin{tabular}{|l|l|l|l|}
\hline Characteristic & Response & Number & Percentage \\
\hline Age group (years) & $15-24$ & 157 & 41.5 \\
\hline & $25-34$ & 195 & 51.6 \\
\hline & $35+$ & 26 & 6.9 \\
\hline Marital status & Never married & 221 & 58.5 \\
\hline & Currently married & 16 & 4.2 \\
\hline & Cohabiting & 64 & 16.9 \\
\hline & Divorced/Widowed & 77 & 20.4 \\
\hline
\end{tabular}




\begin{tabular}{|l|l|l|l|}
\hline Education & None & 19 & 5.0 \\
\hline & Some primary & 250 & 66.2 \\
\hline & Above primary & 109 & 28.8 \\
\hline Parity & Nulliparous & 114 & 30.2 \\
\hline & One & 139 & 36.8 \\
\hline & Two & 87 & 23.0 \\
\hline & More than two & 38 & 10.0 \\
\hline
\end{tabular}

Source of information, access and knowledge on VCT services among bar waitresses

Almost all respondents 376 (99.5\%) were aware of places where they could access VCT services. Of these, 364 (96.8\%) accessed VCT services at health care facilities. However, less than a third (30.3\%) mentioned health care facilities as their main source of information on VCT (Figure 1). Of all study participants, 270 (71.4\%) had high level of knowledge of VCT services. Except one respondent, the rest were able to mention the benefits of VCT services with majority mentioning the quest to establish one's HIV status followed by strive to self-protect against acquiring HIV infection.

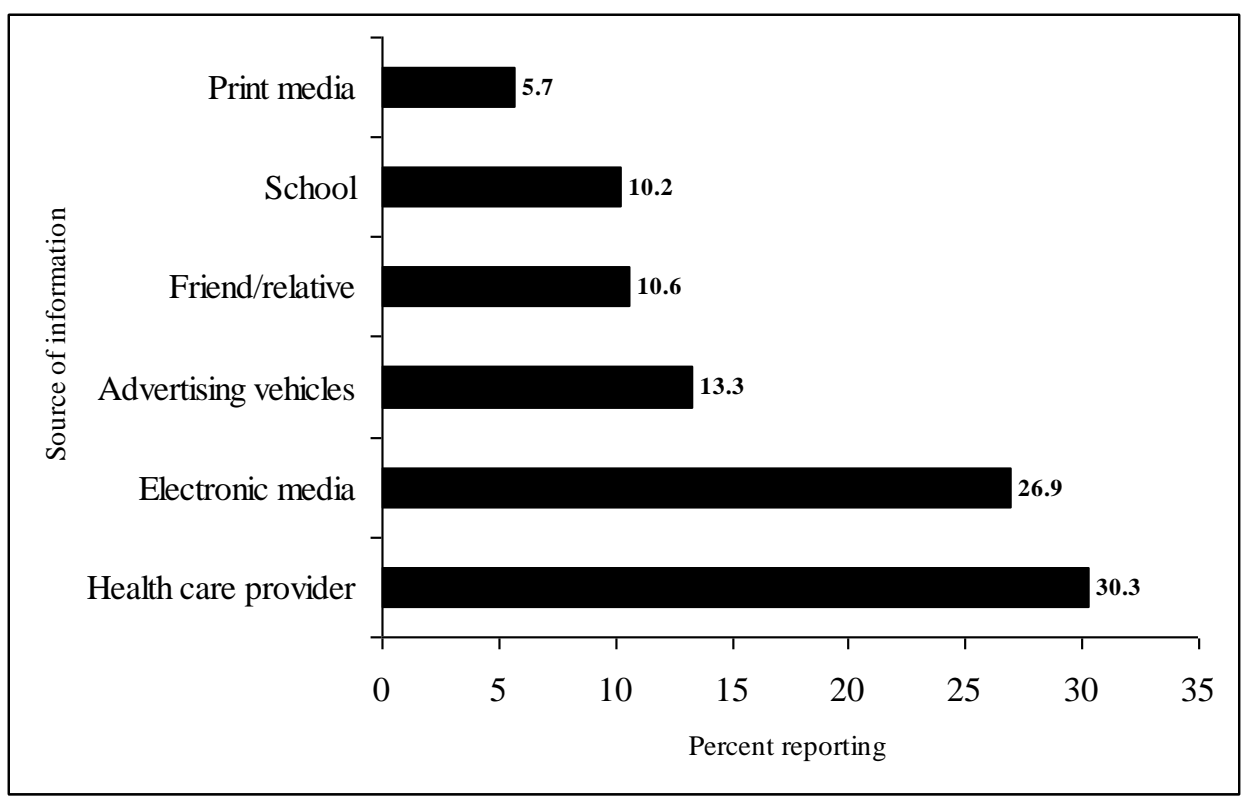

Figure 1: Main source of information on VCT services

\section{Utilization of VCT services}

While majority of the bar waitresses 339 (89.7\%) affirmed to have ever utilized VCT services at some point in their lifetime, less than half of them, $172(45.5 \%)$ reported to have recently utilized VCT services (within the past six months). In the bivariate analysis, variables that had p-values less than 0.2 were education, marital status, and level of knowledge of VCT, condom use, parity, practicing anal sex, lack of fear of stigma and lack of fear of testing positive for HIV. Therefore, these variables were fitted in the binary logistic regression model to predict utilisation of VCT services. 
Table 2. Multivariable binary logistic regression analysis for factors associated with VCT utilisation among bar waitresses

\begin{tabular}{|c|c|c|c|}
\hline \multirow{2}{*}{ Variable } & \multirow[b]{2}{*}{$\begin{array}{l}\text { Number (\%) utilizing } \\
\text { VCT services }\end{array}$} & \multicolumn{2}{|l|}{ OR $(95 \% \mathrm{Cl})^{*}$} \\
\hline & & Unadjusted & Adjusted \\
\hline \multicolumn{4}{|l|}{ Education level } \\
\hline No education & $14(73.7)$ & Reference & Reference \\
\hline Some primary & $226(90.4)$ & $3.4(1.1,10.1)$ & $2.8(0.6,14.3)$ \\
\hline Above primary & $99(90.8)$ & $3.5(1.1,11.9)$ & $4.6(0.8,27.9)$ \\
\hline \multicolumn{4}{|l|}{ Marital status } \\
\hline Never married & $186(84.2)$ & Reference & Reference \\
\hline Ever 'married' $\dagger$ & $153(97.5)$ & $2.5(0.9,7.3)$ & $1.1(0.3,3.6)$ \\
\hline \multicolumn{4}{|l|}{ Parity } \\
\hline At least one & $256(97.0)$ & $11.9(5.3,27.0)$ & $14.5(5.8,36.0)$ \\
\hline Nulliparous & $83(72.8)$ & Reference & Reference \\
\hline \multicolumn{4}{|l|}{ VCT knowledge } \\
\hline Low/Moderate & $85(79.4)$ & Reference & Reference \\
\hline High & $254(94.1)$ & $4.3(2.2,8.5)$ & $7.3(2.8,19.2)$ \\
\hline \multicolumn{4}{|l|}{ Condom use (recent act) } \\
\hline Yes & $181(92.3)$ & $1.7(0.8,3.3)$ & $3.2(1.4,7.0)$ \\
\hline No & $158(87.8)$ & Reference & Reference \\
\hline \multicolumn{4}{|l|}{ Ever practiced anal sex } \\
\hline Yes & $32(82.1)$ & $0.5(0.5,1.2)$ & $1.0(0.3,3.7)$ \\
\hline Never & $307(90.6)$ & Reference & Reference \\
\hline \multicolumn{4}{|c|}{ Fear of testing HIV positive } \\
\hline Yes & $205(87.6)$ & Reference & Reference \\
\hline No & $134(93.7)$ & $2.0(1.0,5.0)$ & $5.0(1.7,10.0)$ \\
\hline \multicolumn{4}{|l|}{ Fear of being stigmatized } \\
\hline Yes & $160(87.4)$ & Reference & Reference \\
\hline No & $179(92.3)$ & $1.7(0.9,3.4)$ & $3.1(1.2,8.1)$ \\
\hline
\end{tabular}

* Odds Ratio (95\% Confidence Interval); † Married, cohabiting, divorced or widowed

Independent factors which remained significantly associated with VCT utilisation among bar waitresses included parity, knowledge level of VCT, condom use in the recent sexual act, not fearing testing positive for HIV and not fearing stigmatization. Bar waitresses with a high level of knowledge about VCT services had more than seven-fold odds to utilize VCT services as compared to those with low or moderate knowledge $(\mathrm{OR}=7.3 ; 95 \% \mathrm{Cl}=2.8,19.2)$ independent of other factors. Bar waitresses with at least one child had more than 14 odds to utilize VCT services than those without a child (OR = $14.5 ; 95 \% \mathrm{Cl}=5.8,36.0)$ independent of other factors. Similarly, waitresses who reported to have no fear of testing positive for HIV had five odds to utilize VCT services as compared to those scared of testing positive for $\mathrm{HIV}(\mathrm{OR}=5.0 ; 95 \% \mathrm{Cl}=1.7,10.0)$ independent of other factors (Table 2 ).

\section{Discussion}

In this study, most of the bar waitresses reported to have ever tested for HIV. Such high rates of testing are anticipated due to numerous sensitization campaigns, which encourage people to know their HIV status. The increase in the number of HIV VCT centres, coupled with availability of antiretroviral treatment, which has played a pivotal role in increasing the uptake of VCT services, might have also 
contributed to the observed high rates of HIV testing. The reported VCT utilisation among the bar waitresses in this study is higher than that documented for the general population in Tanzania (THMIS, 2013). The difference could have been contributed by the nature of the working environment of the waitresses, which puts them at an increased risk of engaging in high-risk sexual behaviours. However, utilisation of VCT services in the past 6 months decreased drastically from $90 \%$ to $46 \%$, affirming to earlier observations that HIV testing is not done at least once per year as recommended especially for members of perceived high-risk groups (NACP, 2011).

Studies on VCT utilisation elsewhere (Maman et al., 2001; Kasote \& Tsue, 2005; McGarrigle et al., 2005; DeGraft-Johnson et al., 2005; Addis et al., 2013; Tuntufye, 2014) have reported that age, education level and marital status are associated with utilisation of VCT services. In our study, neither education level nor marital status of the respondents remained independent predictor of utilisation of VCT services in the multivariate analysis. On the other hand, having at least one child, high level of knowledge on VCT, using a condom in the most recent sexual act as well as lack of fear of positive results and stigmatization increased the likelihood of utilizing VCT services. However, the present study was done in an urban area with high utilisation of antenatal and facility delivery services, and hence some respondents were likely exposed to VCT and subsequent counselling. The significant association between condom use and utilisation of VCT services in the present study conforms to what has been reported in Vietnam (Train et al., 2013). It is plausible that respondents who use condoms are more confident with their HIV status and therefore have less fear of testing positive. Bar waitresses who used condom in the last sexual encounter most likely have higher knowledge of HIV/AIDS, the role of condom in preventing HIV infection as well as the relevance of HIV testing in infection prevention.

This study has two potential limitations. Firstly, the sample size was limited to only bar waitresses working in one district of Dar es Salaam, restricting generalization of inferences to the other districts. Secondly, the study tool was not self-administered because of possibility of illiteracy among respondents. The use of interviewers may potentially lead respondents to offer socially desirable response. An anonymous data collection tool that gave confidence to the waitresses mitigated this. Despite its methodological limitations, our findings provide important insights on the utilisation of VCT services among a high-risk population subgroup that has not been adequately involved in HIV-related research. The findings highlight the need to design strategies that will enhance effective utilisation of VCT services among bar waitresses.

\section{Competing interests}

The authors declare that they have no competing interests.

\section{Acknowledgements}

The authors are grateful to the School of Public Health and Social Sciences, Muhimbili University of Health and Allied Sciences, through the CDC program, for sponsoring data collection leading to this publication. The funders had no role in the study neither in the decision to publish. Deep appreciations go to all bar waitresses who volunteered the valuable information and to all data enumerators.

\section{References}

Addis, Z., Yalew, A., Shiferaw, Y., Alemu, A., Birhan, W., Mathewose, B. \& Tachebele, B. (2013). Knowledge, attitude and practice towards voluntary counseling and testing among university students in North West Ethiopia : a cross sectional study. BMC Public Health 13(714): 221-229. 
Aho, J., Nguyen, V.-K., Diakite, S., Sow, A. \& Rashed, S. (2011) High acceptability of HIV voluntary counselling and testing among female sex workers : impact of individual and social factors. HIV Medicine 1-10.

Charles, M.P., Kweka, E.J., Mahande, A.M., Barongo, L.R., Shekalaghe, S., Nkya, H.M., Lowassa, A. \& Mahande, M. J. (2009) Evaluation of uptake and attitude to voluntary counseling and testing among health care professional students in Kilimanjaro region, Tanzania. BMC Public Health, 9, 128. http://doi.org/10.1186/1471-2458-9-128

DeGraft-Johnson, J., Paz-Soldan, V., Kasote, A. \& Tsui, A. (2005) HIV Voluntary Counseling and Testing Service Preference in a Rural Malawi Population. AIDS and Behavior 9(4): 475-484.

Kapiga, S.H., Sam, N.E., Shao, J.F., Renjifo, B., Masenga, E.J., Kiwelu, I.E., Manongi, R., Fawzi, W. \& Essex, M. (2002) HIV-1 epidemic among female bar and hotel workers in northern Tanzania: risk factors and opportunities for prevention. Journal of Acquired Immune Deficiency Syndromes 29(4): 409-417.

Maman, S., Mbwambo, J., Hogan, N., Kilonzo, G., \& Sweat, M. (2001). Women's barriers to HIV-1 testing and disclosure: challenges for HIV-voluntary counseliing and testing. AIDS Care, 13(5), 595-603.

McGarrigle, C.A., Mercer, C.H., Fenton, K.A., Copas, A.J., Wellings, K., Erens, B. \& Johnson, A.M. (2005). Investigating the relationship between HIV testing and risk behaviour in Britain : National Survey of Sexual Attitudes and Lifestyles 2000. AIDS 19(1): 77-84.

NACP (2008) HIV and AIDS Voluntary Counselling and Testing Participant's Manual. National AIDS Control Programme, Dar es Salaam, Tanzania.

NACP. (2011) HIV Behavioral and Biological Surveillance Survey Among Female Sex Workers in Dar es Salaam, 2010. Dar es Salaam, Tanzania. Retrieved from http://ihi.eprints.org/2793/1/NACP_Report_on_the_HIV_FSW.pdf

Obermeyer, C.M. \& Osborn, M. (2007) The utilization of testing and counseling for HIV: A review of the social and behavioral evidence. American Journal of Public Health 97: 1762-1774.

Riedner, G., Rusizoka, M., Hoffmann, O., Nichombe, F., Lyamuya, E., Mmbando, D., Maboko, L., Hay, P., Todd, J., Hayes, R., Hoelscher, M. \& Grosskurth, H. (2003) Baseline survey of sexually transmitted infections in a cohort of female bar workers in Mbeya Region, Tanzania. Sexually Transmitted Infections 79: 382-387.

THMIS (2008) Tanzania HIV/AIDS and Malaria indicator Survey 2007-08. Tanzania Commission for AIDS (TACAIDS), Zanzibar AIDS Commision (ZAC), National Bureau of Statistics (NBS), Office of the Chief Government Statistician (OCGS), and Macro International Inc. 2008.

THMIS (2013) Tanzania HIV/AIDS and Malaria Indicator Survey 2011-12. Tanzania Commission for AIDS (TACAIDS), Zanzibar AIDS Commision (ZAC), National Bureau of Statistics (NBS), Office of the Chief Government Statistician (OCGS), and ICF International 2013.

Tran, T., Nguyen, L.T., Nguyen, N. \& Pan, H.T. (2013) HIV voluntary testing and perceived risk among female sex workers in the Mekong Delta Region of Vietnam. Global Health Action 6: 1-7.

Tuntufye, S.M. (2014) Education level and human immunodeficiency virus (HIV)/acquired immune deficiency syndrome (AIDS) knowledge in Kenya. Journal of AIDS and HIV Research 6: 28-32.

UNAIDS (2017) Fact sheet - Latest global and regional statistics on the status of the AIDS epidemic. Unaids. Retrieved

from http://www.unaids.org/sites/default/files/media_asset/UNAIDS_FactSheet_en.pdf

Uzochukwu, B., Uguru, N., Ezeoke, U., Onwujekwe, O. \& Sibeudu, T. (2011). Voluntary counseling and testing (VCT) for HIV/AIDS: A study of the knowledge, awareness and willingness to pay for VCT among students in tertiary institutions in Enugu State Nigeria. Health Policy 99: 277-284.

Vallely, A., Shagi, C., Kasindi, S., Desmond, N., Lees, S., Chiduo, B., Hayes, R., Allen, C., Ross, D. \& Mlcrobicides Development Programme (2007) The benefits of participatory methodologies to develop effective community dialogue in the context of a microbicide trial feasibility study in 
Mwanza, Tanzania. BMC Public Health 7(1): 133.

Watson-Jones, D., Baisley, K., Weiss, H.A., Tanton, C., Changalucha, J., Everett, D., Chirwa, T., Ross, D., Clayton T. \& Hayes, R. (2007) Risk Factors for Herpes Simplex Virus Type 2 and HIV Among Women at High Risk in Northwestern Tanzania. Journal of Acquired Immune Deficiency Syndrome 46: 631642. 